\title{
Modelos não-lineares para a estimativa da produção de tomate do tipo cereja
}

\author{
Nonlinear models for estimating cherry tomato yield
}

\author{
Alessandro Dal'Col Lúcio ${ }^{\mathrm{I}}$ Bruno Giacomini Sari' ${ }^{\mathrm{II}}$ Maristela Rodrigues ${ }^{\mathrm{II}}$ \\ Ledur Macht Bevilaqua ${ }^{\mathrm{III}}$ Higor Modesto Garlet Voss ${ }^{\mathrm{III}}$ Diego Copetti ${ }^{\mathrm{II}}$ Maurício Faé ${ }^{\mathrm{II}}$
}

RESUMO

Os objetivos do trabalho foram ajustar modelos de regressão não-linear para a estimativa da produção de frutos de tomate tipo cereja e identificar o tamanho da parcela com melhor poder de explicação e ajuste dos modelos, utilizando dois ensaios de uniformidade em estufa plástica. Os modelos ajustados foram o logístico e o de von Bertalanffy, para peso médio de frutos, número médio de frutos e de cachos acumulado nas múltiplas colheitas e com diferentes tamanhos de parcela. Os modelos apresentaram estimativas semelhantes entre si e as mesmas estimativas dos parâmetros em todos os casos de tamanho de parcela. Os modelos de regressão não-linear estudados descrevem satisfatoriamente o comportamento da produção de frutos de tomate tipo cereja cultivado em estufa plástica. Parcela constituída de duas plantas para ensaios em estufa plástica de $250 \mathrm{~m}^{2}$ e de três plantas em estufa plástica de $200 \mathrm{~m}^{2}$ proporcionam bom poder de explicação nas estimativas dos parâmetros dos modelos.

Palavras-chave: Lycopersicon esculentum var. cerasiforme, ambiente protegido, modelos de crescimento, modelo logístico, modelo de von Bertalanffy, olericultura.

\section{ABSTRACT}

The aim of this paper was to test nonlinear regression models for estimating the cherry tomato production and to identify the plot size with better fitting quality and adjustment of the models, using two uniformity trials in greenhouse. The logistic and von Bertalanffy models were used on variables, as the means fruit weight, the means number of fruits and bunches accumulated in the multiple harvests and with different plot sizes. The models presented similar estimates for all parameters in all plot sizes studied. The nonlinear models adequately describe the behavior of the production of cherry tomato fruits cultivated in greenhouse. Plots constituted by two and three plants, respectively in trials with greenhouse of $250 \mathrm{~m}^{2}$ and $200 \mathrm{~m}^{2}$, provide a good fitting quality in the models adjustment.

Key words: Lycopersicon esculentum var. cerasiforme, protected environment, growth models, logistic model, von Bertalanffy model, horticulture.

\section{INTRODUÇÃO}

A variabilidade presente nos cultivos olerícolas é elevada, especialmente em ambiente protegido, em virtude de fatores como manejo cultural mais intensivo em relação às outras culturas, proximidade das linhas de cultivo com as laterais da estufa plástica, presença ou ausência de frutos aptos a serem colhidos em determinada colheita, ponto de colheita subjetivo, manejo cultural mais intensivo em relação às outras culturas e as múltiplas colheitas realizadas em algumas culturas (LOPES et al., 1998; LORENTZ et al., 2005; LUCIO et al., 2008; CARPES et al., 2010; HAESBAERT et al., 2011).

A relação entre as variáveis observadas em experimentos com olerícolas e seus comportamentos durante o ciclo produtivo da espécie é importante, pois gera informações de como devem ser planejadas e realizadas as múltiplas colheitas, no sentido de reduzir o número de observações com valores zero para as variáveis número e peso de frutos e de cachos colhidos. Uma estratégia interessante neste sentido é

\footnotetext{
IDepartamento de Fitotecnia, Centro de Ciências Rurais (CCR), Universidade Federal de Santa Maria (UFSM), 97105-900, Santa Maria, RS, Brasil. E-mail: adlucio@ufsm.br. Autor para correspondência.

"Programa de Pós-graduação em Agronomia, CCR, UFSM, Santa Maria, RS, Brasil.

IIICurso de Agronomia, CCR, UFSM, Santa Maria, RS, Brasil. 
estimar o tamanho ideal da parcela que proporcione a menor variância entre as diferentes parcelas avaliadas, pois, muitas vezes, os pesquisadores resolvem esse problema empiricamente, usando tamanhos práticos, no sentido da condução do experimento, da área disponível ou de sua experiência. Outra estratégia é o acúmulo dos valores dessas variáveis em cada planta. Com este acúmulo, gera-se um comportamento crescente nos valores observados em cada uma das plantas avaliadas, possibilitando, assim, a aplicação de técnicas de análise de regressão na estimativa dos valores das variáveis já mencionadas.

Dentre os modelos de regressão, aqueles classificados como não-lineares são úteis para descrever o crescimento de indivíduos ao longo do tempo, pois facilitam a tomada de decisão do pesquisador, por apresentarem parâmetros com interpretação biológica. Os modelos não-lineares geralmente são adotados quando se suspeita que a relação entre a variável resposta e os preditores segue a uma função particular (SEBER \& WILD, 1989; SMYTH, 2002). Já DRAPER \& SMITH (1981) afirmam que o tipo de modelo não-linear adotado depende da área de pesquisa, do problema específico e do tipo de crescimento que se quer modelar. Também relatam que os modelos de crescimento não-lineares são aplicados em diversas áreas do conhecimento, como biologia, ciências econômicas e políticas, engenharia química, entre outros, indicando a vasta possibilidade de aplicação desta técnica de análise estatística para descrever as relações entre diferentes variáveis.

$\mathrm{Na}$ área das ciências agrárias, de forma particular na olericultura, as aplicações de modelos de crescimento não-lineares são no sentido de avaliar todo o ciclo de uma espécie específica ou de modelar o crescimento de acordo com a aplicação de diferentes manejos culturais, como, por exemplo, os trabalhos de MARTINS FILHO et al. (2008) e de VIEIRA NETO et al. (2013). Entretanto, não foram identificados trabalhos que descrevessem as relações nãolineares da produção de frutos com o avançar do ciclo produtivo das espécies olerícolas com múltiplas colheitas e com dados apresentando uma alta variabilidade.

Assim, os objetivos deste trabalho foram ajustar modelos de regressão não-linear para a descrição da produção de frutos de Lycopersicon esculentum var. cerasiforme e identificar o tamanho da parcela que proporcione o melhor poder de explicação e ajuste dos modelos.

\section{MATERIAL E MÉTODOS}

Foram realizados dois ensaios de uniformidade, sem a aplicação de tratamentos, com o híbrido de tomate do tipo cereja 'Lili' na estação primavera/verão de 2013, no Departamento de Fitotecnia da Universidade Federal de Santa Maria (latitude $29^{\circ} 43$ 'S, longitude $53^{\circ} 43$ 'W e altitude $95 \mathrm{~m})$, em estufas plásticas do tipo arco pampeano cobertas com filme de polietileno de baixa densidade (PEBD), com espessura de 150 micras e aditivo antiUV, orientadas no sentido norte-sul e com dimensões distintas. Uma estufa apresenta pé direito de $3,00 \mathrm{~m}$ e $4,00 \mathrm{~m}$ na parte central, com $20,00 \mathrm{~m}$ de comprimento e $10,00 \mathrm{~m}$ de largura, totalizando uma área de $200 \mathrm{~m}^{2}$; a segunda estufa apresenta pé direito de $4,50 \mathrm{~m}$ e $5,50 \mathrm{~m}$ na parte central, com $25,00 \mathrm{~m}$ de comprimento e $10,00 \mathrm{~m}$ de largura, totalizando uma área de $250 \mathrm{~m}^{2}$.

No mês anterior à instalação do experimento, o pH do solo foi elevado a 6,5 e os níveis de fósforo a $300 \mathrm{mg} \mathrm{dm}^{-3} \mathrm{em}$ ambas as estufas. O calcário e o fósforo utilizados na correção do solo foram incorporados com uma enxada rotativa, sendo que posteriormente foram erguidos camalhões com aproximadamente $0,20 \mathrm{~m}$ de altura e $0,30 \mathrm{~m}$ de largura, cobertos com faixas de "mulching" de filme opaco de PEBD de cor preta. Em cada estufa, foram erguidos oito camalhões espaçados em $1 \mathrm{~m}$ entre si. $\mathrm{O}$ transplante das mudas foi realizado no dia 26 de outubro de 2013 em ambas as estufas. O espaçamento entre plantas foi de $0,5 \mathrm{~m}$ e a adubação foi realizada com $150 \mathrm{~kg} \mathrm{ha}^{-1}$ de N, 250 $\mathrm{kg} \mathrm{ha}^{-1}$ de P e $125 \mathrm{~kg} \mathrm{ha}^{-1}$ de K. Foram realizadas duas adubações de cobertura com $30 \mathrm{~kg} \mathrm{ha}^{-1}$ de N, $15 \mathrm{~kg} \mathrm{ha}^{-1}$ de P e $30 \mathrm{~kg}$ $\mathrm{ha}^{-1}$ de $\mathrm{K}$ em um intervalo de 23 dias, sendo que, após a segunda adubação de cobertura, foi realizado o tutoramento das plantas em duas hastes, sendo eliminados os brotos laterais. A cada 14 dias, foram realizadas pulverizações foliares de cálcio e boro com o objetivo de evitar o "fundo preto" nos frutos de tomate. Também, a cada 14 dias, foram realizadas aplicações de Dithane ${ }^{\circledR}$ (dose de $3 \mathrm{~kg} \mathrm{ha}^{-1}$ ) e Recop ${ }^{\circledR}$ (dose de $200 \mathrm{~g}$ por 100L de água) para controle preventivo de doenças

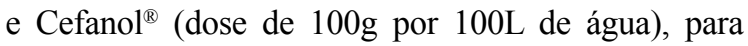
controle preventivo de pragas.

Cada planta foi considerada uma unidade experimental básica (UB) e, devido ao comprimento diferenciado, o número de UB's nas linhas de cultivo foi distinto entre as estufas plásticas, sendo $40 \mathrm{UBs}$ na de $250 \mathrm{~m}^{2}$ e $30 \mathrm{UBs}$ na de $200 \mathrm{~m}^{2}$. Todos os cachos de todas as UB's foram colhidos, sendo considerado o cacho apto para colheita aquele em que a maioria dos frutos apresentavam mudança de coloração de verde para avermelhado. 
Foram realizadas três colheitas, aos 66 , 82, 101 dias após o transplante (DAT) na estufa plástica de $250 \mathrm{~m}^{2}$ e, naquela de $200 \mathrm{~m}^{2}$, aos 75 , 88, 103 DAT. Em cada colheita realizada, foram observadas, em cada UB, as variáveis peso de frutos (em gramas), usando balança digital com precisão de $0,01 \mathrm{~g}$, número de frutos e número de cachos colhidos. Foram construídos gráficos boxplot para os valores individuais e acumulados para cada colheita, no intuito de identificar a variabilidade existente no banco de dados. Após, foram simulados diferentes tamanhos de parcela, em agrupamentos de UB, no sentido da linha de cultivo, pelos múltiplos do número de unidades básicas total por linha, de modo a utilizar toda a área experimental. Assim, foram simuladas parcelas com 1, 2, 4, 5, 8, 10 e 20 UBs na estufa de $250 \mathrm{~m}^{2}$ e parcelas de $1,2,3,5,6,10$ e 15 UBs na estufa de $200 \mathrm{~m}^{2}$. A partir dessas situações, foram estimados o peso médio de frutos, número médio de frutos e número médio de cachos colhidos por parcela, por agrupamento de UB dentro de cada uma das múltiplas colheitas e o total acumulado das mesmas variáveis com o decorrer do ciclo produtivo da cultura.

Foi realizado o teste de Levene para a identificação da homogeneidade dos resíduos entre as múltiplas colheitas realizadas e o teste de DurbinWatson para a identificação da autocorrelação entre os resíduos. Nos ajustes dos modelos de regressão nãolineares, para descrever o comportamento acumulado das variáveis observadas com a evolução do ciclo produtivo, foram adotados os modelos Logístico (SEBER \& WILD, 1938): $Y_{i}=\frac{\beta_{1}}{1+e^{\left(\beta_{2}-\beta_{3} X_{i}\right)}}+\varepsilon_{i}$ e e de von Bertalanffy (von BERTALANFFY, 1957): $Y_{i}=\beta_{1}\left(1-\beta_{2} e^{\left(-\beta_{3} X_{i}\right)}\right)^{3}+\varepsilon_{i}$. em que: $Y_{i}=$ peso médio acumulado (gramas por planta) ou número médio acumulado de frutos ou número médio de cachos colhidos por unidade básica (variável dependente); $X_{i}=$ tempo, em dias, decorridos da semeadura, até a observação da variável (variável independente); $\beta_{1}=$ parâmetro que representa o peso assintótico; $\beta_{2}$ = parâmetro de locação, de escala; $\beta_{3}=$ parâmetro da taxa de crescimento; $\varepsilon_{\mathrm{i}}=$ erro aleatório.

Para as estimativas dos parâmetros dos modelos, foi utilizado o método dos mínimos quadrados ordinários com processo iterativo de Levenberg-Marquardt, com erro tipo Gaussiano, obtendo os intervalos de confiança de cada estimativa dos parâmetros. Para identificar a qualidade do ajuste e o poder de explicação de cada um dos modelos de regressão, foram estimados os respectivos coeficientes de determinação ajustado $\left(\mathrm{R}_{\mathrm{aj}}^{2}\right)$, erro padrão do ajuste
$(\mathrm{EPA}=\sqrt{Q M \text { Re siduo }})$, os gráficos dos resíduos e o número de iterações para cada modelo.

Em todas as análises estatísticas realizadas foi adotado o nível de 5\% de erro.

\section{RESULTADOS E DISCUSSÃO}

A variabilidade do peso e do número de frutos e de cachos colhidos em cada uma das múltiplas colheitas realizadas apresentou um comportamento diferente nos dois ensaios. Naquele com área de $250 \mathrm{~m}^{2}$, ocorreu uma maior variabilidade na colheita intermediária, enquanto que, no ensaio de $200 \mathrm{~m}^{2}$, a de maior variabilidade foi a primeira colheita, apresentando ainda um comportamento decrescente com o passar do ciclo produtivo (Figura 1). Esperavase que as primeiras e últimas colheitas apresentassem as maiores estimativas da variância, em virtude de serem as que representam o início e o final do ciclo produtivo da cultura, respectivamente, e geralmente apresentarem um maior número de unidades básicas (UBs), sem a presença de frutos aptos a serem colhidos, devido aos menores potenciais de produção de frutos nessas fases.

Esses resultados evidenciam que o manejo de colheita deve ser realizado individualmente, colheita a colheita, de tal forma a reduzir a variabilidade nos dados. A definição adequada do ponto de colheita de cada fruto pode ser uma alternativa prática neste sentido, bem como a definição de intervalos de tempo para cada colheita ao invés da definição de um dia específico.

Ao se trabalhar com os totais acumulados do peso, número de frutos e de cachos colhidos, foi possível identificar que, à medida que o ciclo produtivo das espécies avançava, a variabilidade dos dados foi aumentando (Figura 1). Essa situação também não era esperada, pois, com o acúmulo dos valores observados em cada unidade básica, para cada uma das variáveis, esperava-se que a influência dos valores zero fosse minimizada e, por consequência, a dispersão iria ser eliminada do banco de dados. Mesmo sem a colheita de frutos em uma determinada unidade básica, a variabilidade dos dados continuou alta e crescente, pois, nesse caso específico, o valor acumulado da colheita $n$ manteve-se idêntico àquele valor obtido na colheita $n-1$, enquanto que, naquela UB com frutos colhidos, o valor acumulado aumentou e, assim, a variabilidade dos valores acumulados dentro de cada colheita tende a aumentar (Figura1). Entretanto, mesmo com esse comportamento, o teste de Levene evidenciou a homogeneidade entre as variâncias em 
Estufa Plástica de $250 \mathrm{~m}^{2}$

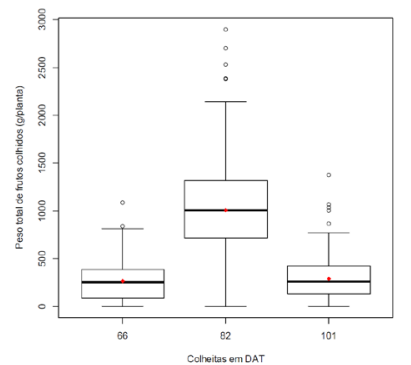

(a)

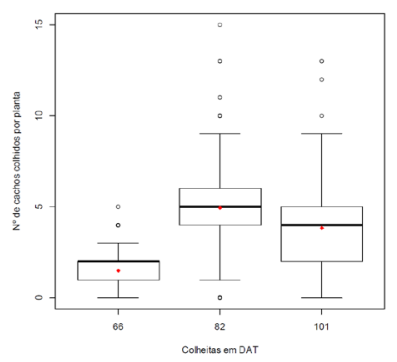

(c)

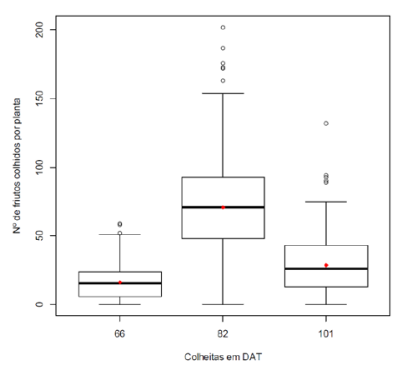

(e)

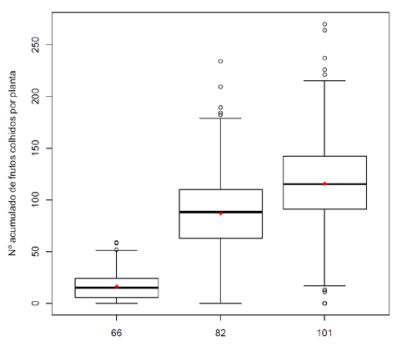

(d)

(f)

Estufa Plástica de $200 \mathrm{~m}^{2}$

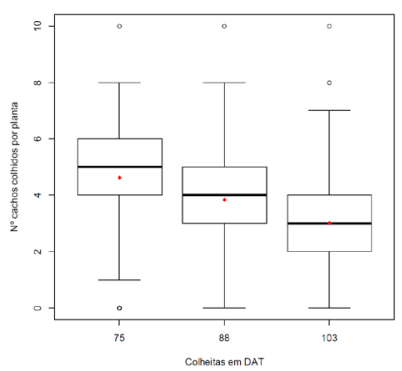

(a)

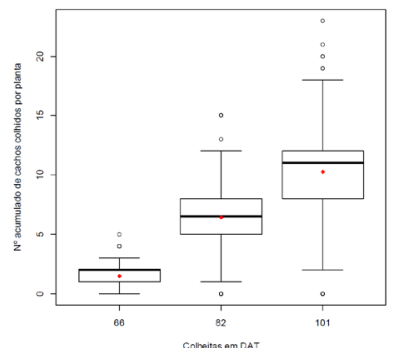

(b)

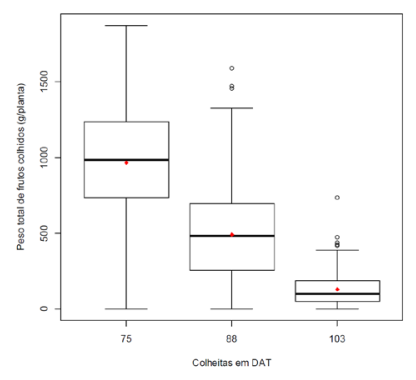

(c)

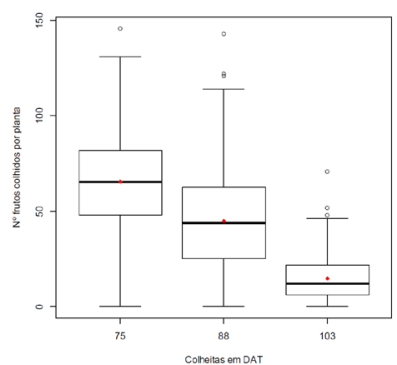

(e)

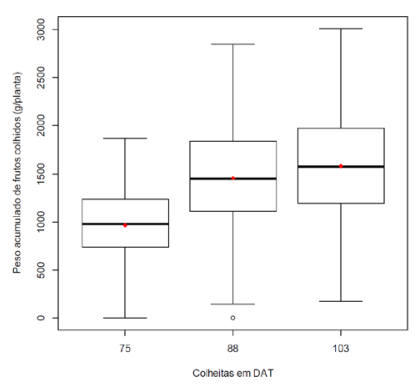

(b)

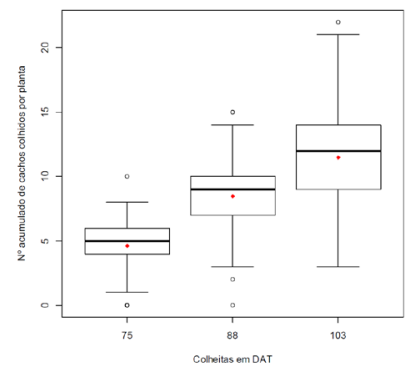

(d)

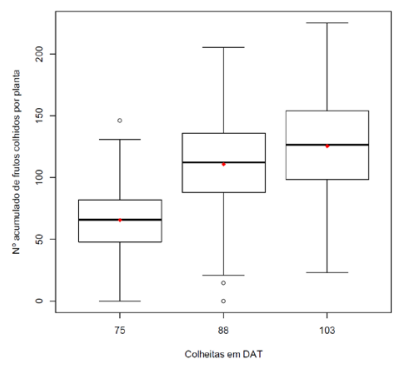

(f)

Figura 1 - Box-plot do peso total (a) e acumulado (b) de frutos (gramas por unidade básica), do número total (c) e acumulado (d) de cachos colhidos por unidade básica e do número total (e) e acumulado (f) de frutos colhidos por unidade básica nos ensaios de uniformidade de Lycopersicon esculentum var. cerasiforme em ensaios de diferentes tamanhos. 
$80 \%$ dos casos e normalidade dos dados em $100 \%$ dos casos analisados. Além disso, foi confirmado o comportamento dos resíduos não correlacionados em todas as situações de tamanho de parcela simulados, com os valores obtidos da estatística do teste de Durbin-Watson variando entre 1,68 e 1,90, indicando a independência dos resíduos em todas as variáveis observadas (GARSON, 2014).

Os ajustes dos modelos de regressão não-linear de crescimento apresentaram sempre as mesmas estimativas dos três parâmetros em todas os casos de tamanho de parcela avaliados, em virtude de serem utilizadas as médias dos valores observados dentro de cada tamanho de parcela. Observou-se também que o modelo de von Bertalanffy apresentou sempre maiores valores de $\hat{\beta}_{1}$ e menores valores de $\hat{\beta}_{3}$, quando comparado com o modelo logístico (Tabela 1), e essas estimativas representam, respectivamente, o peso médio, o número médio de fruto e o número médio de cachos colhidos na fase final do ciclo produtivo e a taxa de crescimento. Assim, os modelos utilizados no estudo descreveram adequadamente o comportamento das variáveis que explicam a produção de frutos de tomate cereja.

À medida que houve o aumento do tamanho da parcela, em número de UBs, observouse que a amplitude do intervalo de confiança de cada uma das estimativas dos parâmetros dos dois modelos ajustados aumentou. Essa situação deu-se em virtude da alteração no valor tabelado da estatística " $t$ " pela redução no número de parcelas dentro de cada linha, com o aumento do tamanho da parcela e, consequentemente, a redução nos graus de liberdade utilizados na construção do intervalo de confiança.
Em relação à qualidade dos ajustes realizados, representados pelos indicadores de qualidade de ajuste, os modelos logístico e de von Bertalanffy apresentaram suas estimativas muito semelhantes (Figuras 2, 3 e 4), exceto no número de iterações no ajuste de cada modelo, onde o logístico apresentou os menores valores, quando comparado com o de von Bertalanffy. Foi identificado que, com o aumento no número de unidades básicas na parcela, houve um aumento nas estimativas do $\mathrm{R}^{2} \mathrm{aj}$, uma redução do erro padrão de ajuste e uma redução nos valores dos resíduos dos modelos, independente da variável observada e da área física do ensaio (Figuras 2, 3 e 4). Assim a estratégia de trabalhar com parcelas de maior tamanho foi eficiente, no sentido de redução da variabilidade existente no banco de dados. Essa situação pode ser explicada pela redução do número de parcelas com valores zero, a partir do aumento no número de unidades básicas que compõem a parcela, reduzindo, por consequência, a variância entre as parcelas dentro de cada colheita realizada.

Em relação ao número de unidades básicas por parcela e adotando o limite mínimo na estimativa do $R_{\text {aj }}^{2} \geq 0,70$, pode-se optar para ensaios em estufas plásticas de $250 \mathrm{~m}^{2}$ adotar parcelas de 2 UBs para avaliar o peso de frutos, o número de frutos e o número de cachos colhidos de tomate cereja. Já para estufas plásticas de $200 \mathrm{~m}^{2}$, as estimativas do $\mathrm{R}^{2}$ aj foram superiores a 0,70 ao adotar parcelas com três ou mais UBs (Figuras 2, 3 e 4). Assim, para uma única recomendação do número de UBs por parcela, é possível planejar experimentos contendo, em cada linha de cultivo, 20 parcelas de duas plantas cada e de

Tabela 1 - Estimativas ( $\hat{\beta}$ ) e respectivos erros padrão (entre parêntesis) dos parâmetros dos modelos logístico e de von Bertalanffy, para o peso médio de frutos, o número médio de frutos e o número médio de cacho de Lycopersicon esculentum var. cerasiforme em ensaios de diferentes tamanhos.

\begin{tabular}{|c|c|c|c|c|c|c|}
\hline \multirow{2}{*}{ Modelos } & \multicolumn{3}{|c|}{-------------------Estufa plástica de 250m²------------------ } & \multicolumn{3}{|c|}{-------------------Estufa plástica de 200m²- } \\
\hline & $\hat{\beta}_{1}$ & $\hat{\beta}_{2}$ & $\hat{\beta}_{3}$ & $\hat{\beta}_{1}$ & $\hat{\beta}_{2}$ & $\hat{\beta}_{3}$ \\
\hline \multicolumn{7}{|c|}{ 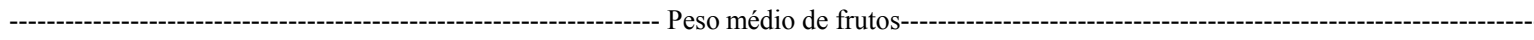 } \\
\hline Logístico & $1560,29(28,049)$ & $14,39(0,847)$ & $0,19(0,012)$ & $1614,03(50,465)$ & $10,58(2,588)$ & $0,15(0,035)$ \\
\hline von Bertalanffy & $1589,04(35,522)$ & $926,68(572,529)$ & $0,11(0,009)$ & $1621,88(57,805)$ & $1428,15(3687,609)$ & $0,12(0,035)$ \\
\hline Logístico & $116,56(2,179)$ & $13,95(0,871)$ & $0,18(0,001)$ & $128,67(3,818)$ & $10,51(1,683)$ & $0,14(0,023)$ \\
\hline von Bertalanffy & $121,14(3,193)$ & $307,92(158,259)$ & $0,10(0,007)$ & $130,15(4,748)$ & $667,93(1102,667)$ & $0,11(0,022)$ \\
\hline Logístico & $10,83(0,214)$ & $11,13(0,675)$ & $0,14(0,009)$ & $13,04(0,608)$ & $7,73(0,785)$ & $0,09(0,011)$ \\
\hline von Bertalanffy & $12,69(0,579)$ & $24,58(8,562)$ & $0,06(0,005)$ & $14,83(1,369)$ & $13,68(9,352)$ & $0,05(0,009)$ \\
\hline
\end{tabular}

Ciência Rural, v.46, n.2, fev, 2016. 


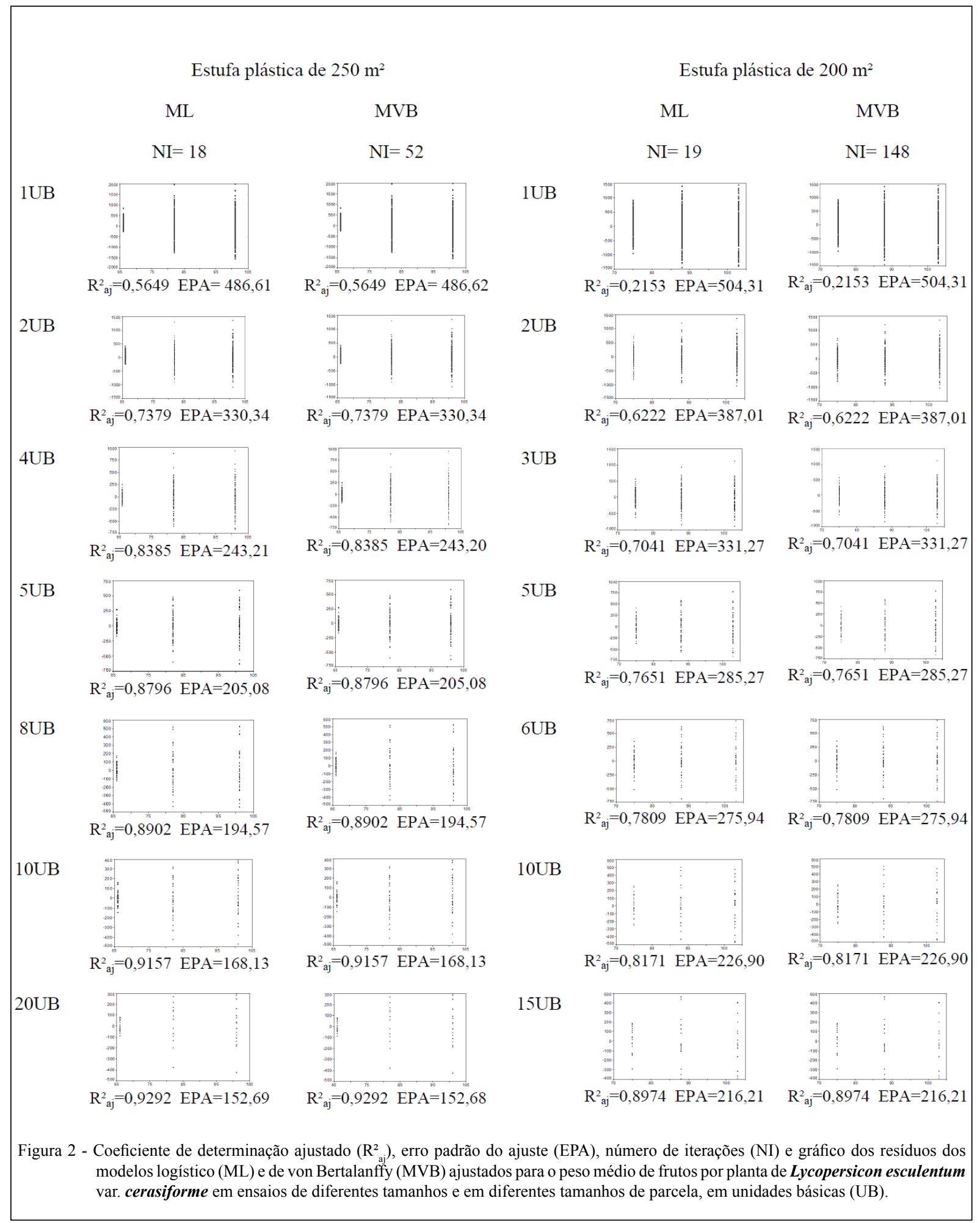

Ciência Rural, v.46, n.2, fev, 2016. 


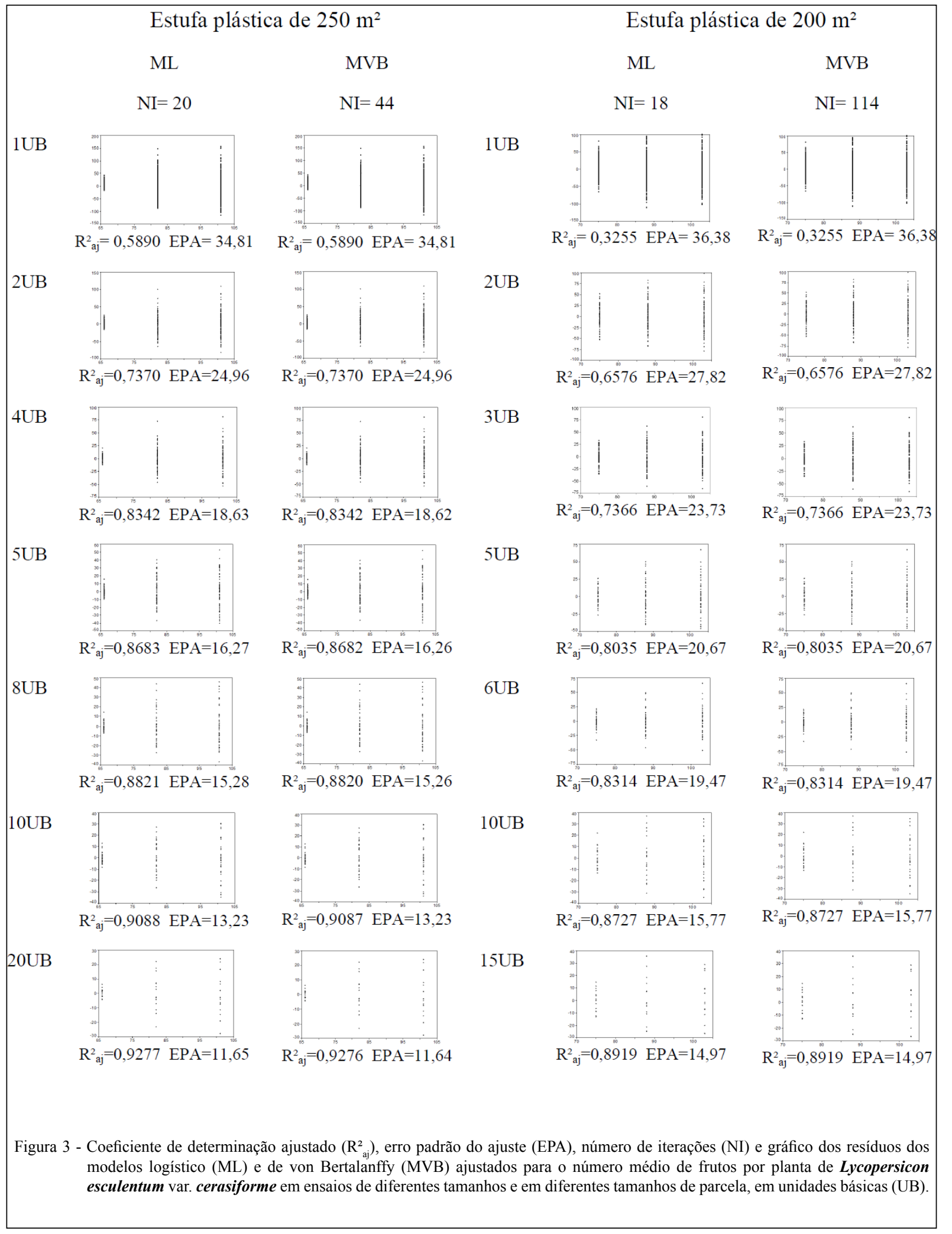

Ciência Rural, v.46, n.2, fev, 2016. 


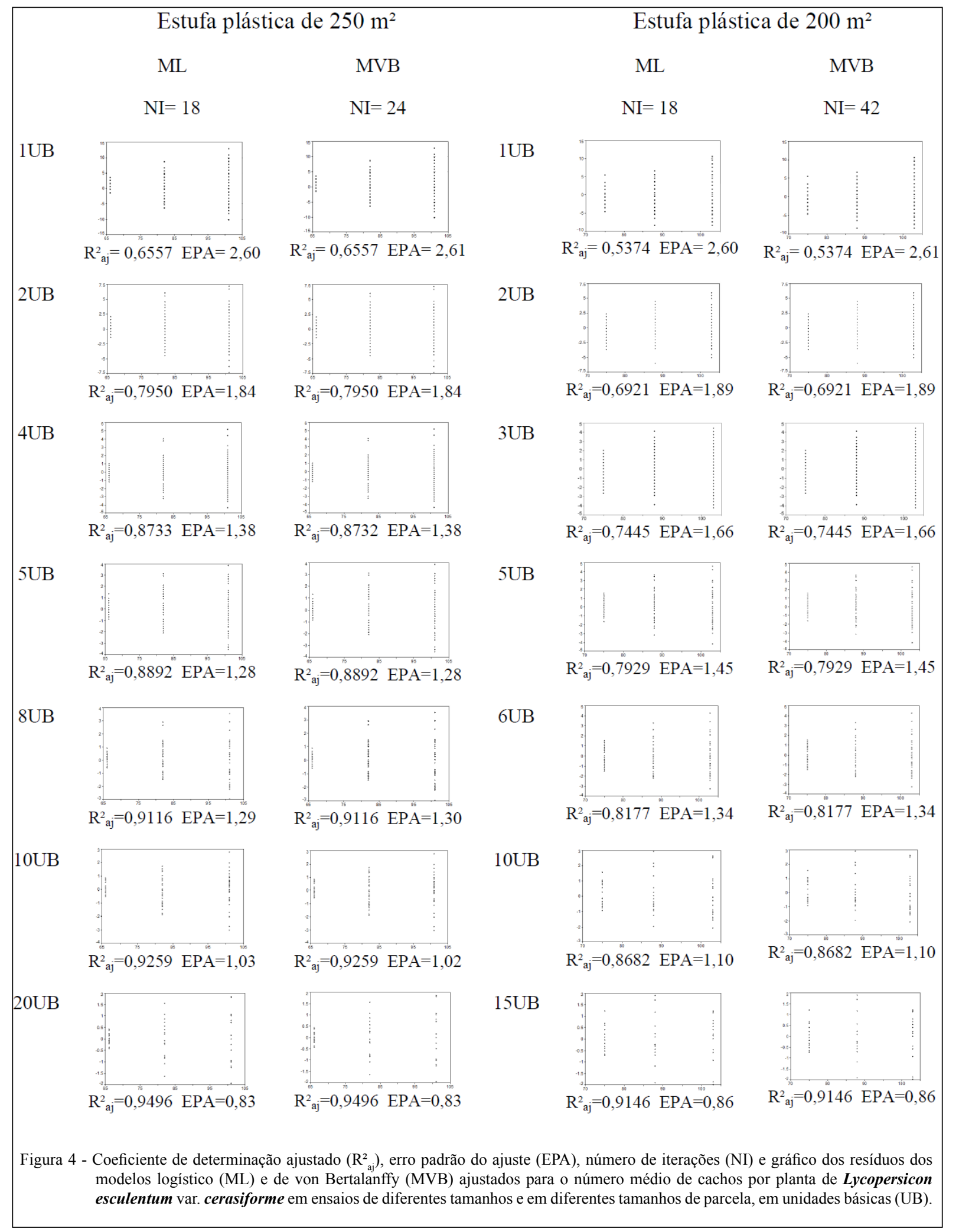

Ciência Rural, v.46, n.2, fev, 2016. 
dez parcelas com três plantas cada, respectivamente, em estufas plásticas de 250 e $200 \mathrm{~m}^{2}$.

\section{CONCLUSÃO}

Os modelos de regressão não-linear de crescimento Logístico e de von Bertalanffy descrevem satisfatoriamente o comportamento do peso médio de frutos, do número médio de frutos e do número médio de cachos colhidos de Lycopersicon esculentum var. cerasiforme, cultivado em estufa plástica.

Parcela constituída de duas plantas no sentido da linha de cultivo, para ensaios com Lycopersicon esculentum var. cerasiforme, em estufa plástica de $250 \mathrm{~m}^{2}$ e de três plantas em estufa plástica de $200 \mathrm{~m}^{2}$, proporcionam bom poder de explicação acima de $70 \%$ nas estimativas dos parâmetros dos modelos Logístico e de von Bertallanfy.

\section{AGRADECIMENTOS}

À Coordenação de Aperfeiçoamento de Pessoal de Nível Superior (CAPES), pela concessão da bolsa de pósdoutorado no exterior, processo n ${ }^{\circ}$ BEX 1457/14-4.

\section{REFERÊNCIAS}

CARPES, R.H. et al. Variabilidade produtiva e agrupamentos de colheitas de abobrinha italiana cultivada em ambiente protegido. Ciência Rural, v.40, p.294-301, 2010. Disponível em: <http://dx.doi.org/10.1590/S0103-84782010005000007>. Acesso em: 10 nov. 2014.

DRAPER, N.; SMITH, H. Applied regression analysis. 2.ed. New York: John Wiley \& Sons, 1981. 709p.

GARSON, G.D. Statnotes: topics in multivariate analysis. Disponível em: <http://faculty.chass.ncsu.edu/garson/PA765/ statnote.htm>. Acesso em: 08 out. 2014.
HAESBAERT, F.M. et al. Tamanho de amostra para experimentos com feijão-de-vagem em diferentes ambientes. Ciência Rural, v.41, p.38-44, 2011. Disponível em: <http://dx.doi.org/10.1590/ S0103-84782011000100007>. Acesso em: 10 nov. 2014.

LOPES S.J. et al. Técnicas experimentais para tomateiro tipo salada sob estufas plásticas. Ciência Rural, v.28, p.193-197. 1998. Disponível em: <http://dx.doi.org/10.1590/S010384781998000200002>. Acesso em: 09 nov. 2014.

LORENTZ, L.H. et al. Variabilidade da produção de frutos de pimentão em estufa plástica. Ciência Rural, v.35, p.316323. 2005. Disponível em: <http://dx.doi.org/10.1590/S010384782005000200011>. Acesso em: 14 nov. 2014.

LÚCIO, A.D. et al. Variância e média da massa de frutos de abobrinha-italiana em múltiplas colheitas. Horticultura Brasileira, v.26, p.335-341, 2008. Disponível em: <http:// dx.doi.org/10.1590/S0102-05362008000300009>. Acesso em: 12 nov. 2014.

MARTINS FILHO, S. et al. Abordagem bayesiana das curvas de crescimento de duas cultivares de feijoeiro. Ciência Rural, v.38, p.1516-1521, 2008. Disponível em: <http://dx.doi.org/10.1590/ S0103-84782008000600004>. Acesso em: 26 nov. 2014.

SEBER, G.A.F.; WILD, C.J. Nonlinear regression. New York: John Wiley \& Sons, 1938. 768p.

SMYTH, G.K. Nonlinear regression. In: EL SHAARAWI; PIEGORSCH, W.W. Encyclopedia of environmetrics. New York: John Wiley \& Sons, 2002. V.3, p.1405-1411.

VIEIRA NETO, J. et al. Produção e curva de crescimento de pepineiros para conserva em manejo convencional e com controle alternativo de pragas. Revista de Ciências Agroveterinárias, v.12, p.229-237, 2013. Disponível em: <http://www.revistas. udesc.br/index.php/agroveterinaria/article/download/5218/3393>. Acesso em: 13 nov. 2014.

von BERTALANFFY, L. Quantitative laws in metabolism and growth. Quarterly Review of Biology, v.32, p.217-231, 1957. Disponível em: <http://www-rohan.sdsu.edu/ jmahaffy/courses/ f14/math636/lectures/allometric/pulse wt vBert 57.pdf $>$. Acesso em: 06 nov. 2014. 